\title{
Stopping Unitary Processes in Fock Space
}

\author{
By
}

\author{
David ApPlebAuM*
}

\begin{abstract}
Let $P^{T}$ be a stop time in symmetric Fock space over $L^{2}\left(\boldsymbol{R}^{+}\right)$. We show that to certain unitary operator valued processes $U=(U(t), t \geqq 0)$ which satisfy a stochastic differential equation driven by "non-commutative noise" in Fock space tensored with an "initial" Hilbert space, we can associate a stopped operator $U(T)$. We use these operators to prove a "stopped cocycle relation" whereby for $P^{T}$ finite the process $(U(T+t), t \geqq 0)$ is factorised as the product of $U(T)$ with a new process $U^{T}=\left(U^{T}(t), t \geqq 0\right)$ beginning "after time $T$ ".
\end{abstract}

\section{§1. Introduction}

In quantum probability, the analogue of a random variable is an operator in some arbitrary von Neumann algebra $\mathcal{A}$ (so that, in general, random variables need not commute) and the appropriate generalisation of a filtration of $\sigma$-algebras is an increasing sequence $\left(\mathcal{A}_{t]}, t \in \boldsymbol{R}^{+}\right)$of von Neumann subalgebras of $\mathcal{A}$. The extension of the notion of stopping time to various examples of this formalism has been discussed by a number of authors ([1], [2], [3]) and is generally agreed to be at the very least, an increasing projection valued process $(P(t), t \geqq 0)$ with each $P(t) \in \mathcal{A}_{t]}$.

We consider the case where $\mathcal{A}=B(\mathscr{H})$ and each $\mathcal{A}_{t]}=B\left(\mathscr{H}_{t]}\right)$ with $\mathscr{H}\left(\mathscr{K}_{t]}\right)$ the symmetric Fock space over $L^{2}\left(\boldsymbol{R}^{+}\right)\left(L^{2}([0, t])\right)$. This example is known to contain a wealth of interesting probabilistic structure (see e.g. [4]) including a generalisation of the Itô-Doob theory of stochastic integration [5]. Building on the work of Hudson in [1], Parthasarathy and Sinha [6] have recently carried out a detailed investigation of stop times within this context.

One of the most significant results obtained in [6] is the strong factorisability of $\mathcal{H}$. For a finite stop time $P^{T}$, it is shown that $\mathscr{H}$ is canonically isomorphic

Communicated by H. Araki, November 28, 1987.

* Department of Mathematics, University Park, Nottingham NG7 2RD England. Forschungszentrum Bielefeld-Bochum-Stochastik, Universität Bielefeld, D-4800 Bielefeld. FRG.

Current address: Department of Mathematics, Statistics \& Operational Research, Trent Polytechnic, Burton Street, Nottingham NG1 4BU England. 
to the tensor product of a "pre- $T$ " Hilbert space $\mathscr{H}_{T}$ and a "post- $T$ " Hilbert space $\mathscr{H}^{T}$ (In [6], a stop time $P^{T}$ is a spectral measure and $T$ is the associated self adjoint operator). The latter space is, in fact, the image of $\mathscr{H}$ under a certain shift isometry which we denote $V^{T}$. The strong Markov property for quantum Brownian motion established in [1] asserts that conjugation of that process by $V^{T}$ yields a new quantum Brownian motion process on $\mathfrak{H}^{T}$.

The purpose of this paper is to study the effects of stopping on certain unitary operator valued processes $U=(U(t), t \geqq 0)$ which were constructed in [5] as the solutions of quantum stochastic differential equations on $h_{0} \otimes \mathscr{H}$ where $h_{0}$ is a complex separable Hilbert space in which initial conditions are specified. The properties of these processes lie at the heart of the application of quantum stochastic calculus to the construction of dilations of quantum dynamical semigroups (see e.g. [5], [7] and [8]). This present work is motivated by the hope that stopping these processes will be of similar value in the formulation and solution of the "quantum Dirichlet problem".

The plan of the paper is as follows. After collecting together some definitions and results in quantum stochastic calculus in $\S 2$, we proceed in $\S 3$ to further develop the main tool of [6], the stop time integral. This enables us to define the stopped operator $U(T)$ associated to the process $U$. In $\S 4$, we show that the operators $U(T+t)$ satisfy a "stopped cocycle relation" thus generalising a well-known result for ordinary "deterministic" time.

We will use the following notation; If $g$ is an interval in $\mathbb{R}^{+}$, then $\mathscr{B}(g)$ denotes the $\sigma$-algebra generated by the Borel sets in $g$. If $T$ is a densely defined, closeable operator on some Hilbert space, the notation $T^{\#}$ will be used whenever the proposition in which it occurs is valid for both $T$ and $T^{*}$.

If $h_{1}$ and $h_{2}$ are Hilbert spaces, the ampliation of an operator $T$ on $h_{1}\left(h_{2}\right)$ is the operator $T \otimes I(I \otimes T)$ on $h_{1} \otimes h_{2}$. For $u \in h_{1}, v \in h_{2}$ we will sometimes, for the sake of simplification, denote the vector $u \otimes v$ in $h_{1} \otimes h_{2}$ by $u v$.

\section{Acknowledgements}

This work was begun at the University of Nottingham while the author was supported by SERC Grant No. GR/D/51292 and completed at Forschungszentrum BiBoS. I would like to thank Robin Hudson for several valuable discussions and K. R. Parthasarathy, K. B. Sinha, P. A. Meyer and M. Evans for stimulating comments on an earlier version of this paper. Finally, my thanks to all at BiBoS for making my stay there so enjoyable. 


\section{§ 2. Preliminaries}

Let $\mathcal{S}$ denote the set of (equivalence classes of) locally bounded functions in $L^{2}\left(\boldsymbol{R}^{+}\right)$. For each $f \in \mathcal{S}, \phi(f)$ will denote the corresponding exponential vector in $\mathscr{A}$. We note that $\{\phi(f), f \in \mathcal{S}\}$ is total in $\mathscr{H}$. We will denote by $\mathscr{H}_{t}$, $\mathscr{H}_{s, t}$ and $\mathscr{H}^{t}$, the symmetric Fock spaces over $L^{2}[0, t], L^{2}[s, t]$ and $L^{2}[t, \infty)$ respectively $\left(s, t \in \boldsymbol{R}^{+}\right.$with $s<t$ ) and write

$$
\begin{array}{cl} 
& h=h_{0} \otimes \mathscr{H} \\
& h_{t}=h_{0} \otimes \mathscr{H}_{t} \\
\text { and } \quad & h_{s, t}=h_{0} \otimes \mathscr{H}_{s, t} .
\end{array}
$$

We identify the spaces $h$ and $h_{s} \otimes \mathscr{H}_{s, t} \otimes \mathscr{H}^{t}$ by means of the canonical isomorphism between them which for $v \in h_{0}, g \in \mathcal{S}$ maps $v \otimes \psi(g)$ to $v \otimes \psi\left(g_{s}\right) \otimes \psi\left(g \chi_{[s, t)}\right)$ $\otimes \psi\left(g^{t}\right)$ where $g_{s}=g \chi_{[0, s)}$ and $g^{t}=g \chi_{[t, \infty)}$.

The shift in $L^{2}\left(\boldsymbol{R}^{+}\right)$is given by

$$
\left(\theta_{s} f\right)(t)=f(t-s) \quad \text { if } s \leqq t
$$

0 otherwise.

We will adopt the convention that $\theta_{\infty}=0$.

For $s \geqq 0$, let $\Gamma\left(\theta_{s}\right)$ be the second quantised shift in $\mathscr{H}$ which we identify with its ampliation to the whole of $h$. Note that for $v \in h_{0}, g \in \mathcal{S}$

$$
\Gamma\left(\theta_{s}\right) v \otimes \psi(g)=v \otimes \psi\left(\theta_{s} g\right) .
$$

$\left\{\Gamma\left(\theta_{s}\right), s \in \boldsymbol{R}^{+}\right\}$is a strongly continuous one parameter semi-group of isometries on $h$. Each $\Gamma\left(\theta_{s}\right)$ has adjoint $\Gamma\left(\theta_{s}^{+}\right)$where

$$
\left(\theta_{s}^{+} f\right)(t)=f(s+t), \quad s, t \in \boldsymbol{R}^{+}, f \in L^{2}\left(\boldsymbol{R}^{+}\right)
$$

We note the relation

for $v \in h_{0}, f \in \mathcal{S}, s \in \boldsymbol{R}^{+}$.

$$
\Gamma\left(\theta_{s}\right) \Gamma\left(\theta_{s}^{\dagger}\right) v \otimes \psi(f)=v \otimes \psi\left(f^{s}\right)
$$

For $\xi \in \mathscr{A}^{t}$, we will use when convenient, the simplified notation $\xi=\psi(0) \xi$, where $\phi(0)$ is understood as the vacuum vector in $\mathscr{H}_{t}$.

Let $A, A^{\dagger}$ and $\Lambda$ denote the annihilation, creation and gauge processes of [5], respectively and let $L, H$ and $W$ be fixed elements of $B\left(h_{0}\right)$ with $L$ arbitrary, $H$ self adjoint and $W$ unitary which we identify with their ampliations in $h$. From [5] and [7], we can assert the existence of a unique unitary operator valued solution $U=(U(t), t \geqq 0)$ to the stochastic differential equation (s.d.e) 


$$
\left.\begin{array}{l}
d U=U\left(L d A^{\dagger}+(W-I) d \Lambda-L^{\dagger} W d A+\left(i H-1 / 2 L^{\dagger} L\right) d t\right) \\
U(0)=I \\
\text { with the following properties }
\end{array}\right\}
$$

(i) Adaptedness

(ii) Localisability

$$
U(t) \in B\left(h_{t}\right), \quad t \in \mathbb{R}^{+}
$$

$$
U(s, t)=U(s)^{*} U(t) \in B\left(h_{s, t}\right), \quad s \leqq t \in \boldsymbol{R}^{+}
$$

(iii) Cocyclicity

$$
U(t)=\Gamma\left(\theta_{s}^{+}\right) U(s)^{*} U(s+t) \Gamma\left(\theta_{s}\right), \quad s, t \in \mathbb{R}^{+} .
$$

Recall that by Theorem 4.1 of [5], we may compute matrix elements of each $U(t), t \in \boldsymbol{R}^{+}$, for $u, v \in h_{0}, f, g \in \mathcal{S}$, by means of the formula

$$
\langle u \otimes \psi(f),(U(t)-I) v \otimes \psi(g)\rangle=\int_{0}^{t}\left\langle u \otimes \psi(f), U(s) K_{f, g}(s) v \otimes \psi(g)\right\rangle d s
$$

where

$$
K_{f, g}(t)=\bar{f}(t) L+\bar{f}(t)(W-I) g(t)-L^{\dagger} W g(t)+i H-1 / 2 L^{\dagger} L \text {. }
$$

Note that each $K_{f, g}(t) \in B\left(h_{0}\right)$ and in particular

$$
\sup _{0 \leqq s \leqq t}\left\|K_{f, g}(s)\right\|<\infty .
$$

We now recall some definitions from [6]. A stop time in $\mathscr{H}$ is a spectral measure $P^{T}$ from $\mathbb{R}^{+} \cup\{\infty\}$ to the space of orthogonal projections in $\mathscr{H}$ such that each $P^{T}([0, t]) \in B\left(\mathscr{H}_{t}\right) \otimes I . \quad s \in \boldsymbol{R}^{+} \cup\{\infty\}$ is said to be a continuity point of $P^{T}$ if $P^{T}(\{s\})=0$. We say that $P^{T}$ is finite whenever $\infty$ is a continuity point. Associated to each finite stop time $P^{T}$ is the (self adjoint) stop time operator $T$ with spectral decomposition $\int_{0}^{\infty} s P^{T}(d s)$.

In the case where $P^{T}$ is finite, a canonical isomorphism was established in [6] between $\mathscr{H}_{\text {f }}$ and the space $\mathscr{H}_{T} \otimes \mathscr{H}^{T}$ where the pre-T subspace $\mathscr{H}_{T}$ is the closure of the linear span of $\left\{\int P^{T}(d s) \phi\left(f_{s}\right) \phi(s), f \in L^{2}\left(\boldsymbol{R}^{+}\right), \phi\right.$ a bounded Borel function on $\left.\mathbb{R}^{+}\right\}$and the post-T subspace $\mathscr{H}^{T}$ is the range of the isometry $V^{T}=$ $\int P^{T}(d s) \Gamma\left(\theta_{s}\right)$. The effect of this isomorphism is to map total vectors in $\mathscr{H}$ of the form $\int P^{T}(d s) \phi\left(f_{s}\right) \phi(s) \Gamma\left(\theta_{s}\right) \eta$ to $\int P^{T}(d s) \psi\left(f_{s}\right) \phi(s) \otimes V^{T} \eta \quad(\eta \in \mathscr{A})$.

It is not difficult to verify that this result remains valid when the vectors $f$ are restricted to lie in $\mathcal{S}$. 
We will denote by $h^{T}$ the space $h_{0} \otimes \mathscr{H}^{T}$.

\section{§3. The Stopped Unitary Process}

In this section, we aim to associate a stopped operator, which we denote $U(T)$, to the process $U=(U(t), t \geqq 0)$ which we described in $\S 2$. Formally, we may thank of $U(T)$ as $\int_{0}^{\infty} U(s) P^{T}(d s)$ (In the terminology of [6], it is $U \circ P^{T}$ ). We proceed by extending the scheme of stop time integration developed in [6].

Let $\mathscr{P}$ be a partition of $[a, b] \subset \boldsymbol{R}^{+} \cup\{\infty\}$ so that $\mathscr{Q}=\left\{a=t_{0}<t_{1}<\cdots<t_{n}<t_{n+1}\right.$ $=b\}$ with each $t_{j}(0 \leqq j \leqq n+1)$ a continuity point of $P^{T}$. We denote by $A^{\uparrow}$ the linear space of (vector-valued) future adapted processes in $\mathscr{H}$ so $\xi \in A^{\uparrow} \Rightarrow \xi=$ $\left(\boldsymbol{\xi}(t), t \in \boldsymbol{R}^{+}\right)$with each $\xi(t) \in \mathscr{H}^{t} . \quad A_{c}^{\uparrow}$ will denote that subspace of $A^{\uparrow}$ comprising those processes $\xi$ for which the map $t \rightarrow \xi(t)$ is continuous from $\boldsymbol{R}^{+}$to $\mathscr{H}$.

For fixed $v \in h_{0}, g \in \mathcal{S}, \xi \in A^{\uparrow}$, we define Riemann sums for the process $U$ by

$$
I_{p}(U, v, g, \xi)=\sum_{j=0}^{n} U\left(t_{j+1}\right) P^{T}\left(\left[t_{\jmath}, t_{j+1}\right]\right) v \otimes \psi\left(g_{t_{j+1}}\right) \otimes \xi\left(t_{j+1}\right)
$$

wherein we have identified $P^{T}$ with its ampliation to the whole of $h$.

Note that by (2.5) and the definition of $P^{T}$, we have the result

$$
P^{T}([s, t]) U\left(y, y^{\prime}\right) P^{T}\left(\left[s^{\prime}, t^{\prime}\right]\right)=0
$$

whenever $s, t, s^{\prime}, t^{\prime}, y$ and $y^{\prime}$ are continuity points of $P^{T}$ with $s<t \leqq y$ and $t \leqq s^{\prime}<t^{\prime} \leqq y^{\prime}$ (cf. Proposition 4.1 of [6]).

We define

$$
\begin{gathered}
\delta(\mathscr{Q})=\max _{0 \leqq j \leqq n}\left(t_{j+1}-t_{j}\right) \\
\omega_{\xi}(\mathscr{P})=\sup \left\{\|\xi(t)-\xi(s)\|^{2}, a \leqq s<t \leqq b, t-s \leqq \delta(\mathscr{L})\right\} \\
\omega^{\prime}(\mathscr{P})=\sup \left\{\int_{s}^{t}|g(\tau)|^{2} d \tau, a \leqq s<t \leqq b, t-s \leqq \delta(\mathscr{L})\right\} \\
M=\sup \left\{\|\xi(s)\|^{2}, a \leqq s \leqq b\right\} .
\end{gathered}
$$

The following result extends Proposition 4.2 of [6].

Proposition 1. Let $\mathscr{P}$ and $\mathscr{Q}^{\prime}$ be partitions of $[a, b]$ such that $\mathscr{Q}^{\prime}$ is finer that $\mathscr{P}$ then

$$
\left\|I_{\mathscr{P}}(U, v, g, \xi)-I_{\mathscr{P}}(U, v, g, \xi)\right\|^{2} \leqq C\left(\omega_{\xi}(\mathscr{P})+\omega^{\prime}(\mathscr{P})+\delta(\mathscr{D})\right)
$$

where $C$ is a constant depending only on $v, g$ and $\xi$.

Proof. Let $\mathscr{Q}$ be as above and take the points of $\mathscr{L}^{\prime}$ which lie between $t_{\text {, }}$ 
and $t_{j+1}$ to be $t_{j_{1}}<t_{j_{2}}<\cdots<t_{j_{n_{j}}}(0 \leqq j \leqq n)$.

We define

$$
t_{j_{0}}=t_{j} \text { and } t_{j_{n_{j+1}}}=t_{j+1} .
$$

We will use the notation

By (3.2) we obtain

$$
E_{j, r}=\left[t_{j_{r+1}}, t_{j}\right] \text { and } \alpha_{j, r}=t_{j+1}-t_{j_{r+1}} .
$$

$$
\begin{aligned}
&\left\|I_{\mathcal{P}^{\prime}}(U, v, g, \xi)-I_{\mathscr{P}}(U, v, g, \xi)\right\|^{2} \\
&=\| \sum_{j} \sum_{r}\left\{U\left(t_{j_{r+1}}\right) P^{T}\left(\left[t_{j_{r}}, t_{j_{r+1}}\right]\right) v \psi\left(g_{t_{j_{r+1}}}\right) \xi\left(t_{j_{r+1}}\right)\right. \\
&\left.\quad-U\left(t_{j+1}\right) P^{T}\left(\left[t_{j}, t_{j+1}\right]\right) v \psi\left(g_{t_{j+1}}\right) \xi\left(t_{j+1}\right)\right\} \|^{2} \\
&=\sum_{j} \sum_{r} \| U\left(t_{j_{r+1}}\right) P^{T}\left(\left[t_{j_{r}}, t_{j_{r+1}}\right]\right) v \psi\left(g_{t_{j_{r+1}}}\right) \xi\left(t_{j_{r+1}}\right) \\
& \quad-U\left(t_{j+1}\right) P^{T}\left(\left[t_{j_{r}}, t_{j_{r+1}}\right]\right) v \psi\left(g_{t_{j+1}}\right) \xi\left(t_{j+1}\right) \|^{2} \\
&=\sum_{j} \sum_{r}\left\|P^{T}\left(\left[t_{j_{r}}, t_{j_{r+1}}\right]\right) \psi\left(g_{j_{j_{r+1}}}\right)\right\|^{2} \times \\
& \quad\left\|v \xi\left(t_{j_{r+1}}\right)-U\left(t_{j_{r+1}}, t_{j+1}\right) v \psi\left(g \chi_{E_{j, r}}\right) \xi\left(t_{j+1}\right)\right\|^{2} \\
& \leqq C_{1} \max _{0 \leqq j \leqq n} \max _{1 \leqq r \leqq n_{j}}\left\|v \xi\left(t_{j_{r+1}}\right)-U\left(t_{j_{r+1}}, t_{j+1}\right) v \psi\left(g \chi_{E_{j, r}}\right) \xi\left(t_{j+1}\right)\right\|^{2}
\end{aligned}
$$

where $C_{1}=e^{\|g\|^{2}}$ and we have written $U\left(t_{j+1}\right)=U\left(t_{j_{r+1}}\right) U\left(t_{j_{r+1}}, t_{j+1}\right)$ and then used

$$
\begin{aligned}
\sum_{j} \sum_{r} \| P^{T} & \left(\left[t_{j_{r}}, t_{j_{r+1}}\right]\right) \psi\left(g_{t_{j+1}}\right) \|^{2} \\
& =\sum_{j} \sum_{r}\left\langle\psi(g), P^{T}\left(\left[t_{j_{r}}, t_{j_{r+1}}\right]\right) \psi(g)\right\rangle e^{-\| g^{t_{j_{r+1}}}} \\
& \leqq C_{1} .
\end{aligned}
$$

Now for each $0 \leqq j \leqq n, 1 \leqq r \leqq n_{j}$

and

$$
\begin{aligned}
& \left\|v \boldsymbol{\xi}\left(t_{j_{r+1}}\right)-U\left(t_{j_{r+1}}, t_{j+1}\right) v \psi\left(g \chi_{E_{j, r}}\right) \xi\left(t_{j+1}\right)\right\|^{2} \\
& \quad \leqq 2\|v\|^{2} \omega_{\xi}(\mathscr{P})+2 M\left\|v \psi(0)-U\left(t_{j_{r+1}}, t_{j+1}\right) v \psi\left(g \chi_{E_{j, r}}\right)\right\|^{2}
\end{aligned}
$$

$$
\begin{aligned}
& \left\|v \psi(0)-U\left(t_{j_{r+1}}, t_{j+1}\right) v \psi\left(g \chi_{E_{j, r}}\right)\right\|^{2} \\
& \quad=\|v\|^{2}+\|v\|^{2} \exp \left\{\int_{E_{j, r}}|g(\tau)|^{2} d \tau\right\}-2 \operatorname{Re}\left\langle v \psi(0), U\left(t_{j_{r+1}}, t_{j+1}\right) v \psi\left(g \chi_{E_{j, r}}\right)\right\rangle .
\end{aligned}
$$

Whence, by (2.6), (2.1) and (2.7) we obtain

$$
\begin{aligned}
& \left\langle v \psi(0), U\left(t_{j_{r+1}}, t_{j+1}\right) v \psi\left(g \chi_{E_{j, r}}\right)\right\rangle \\
& =\left\langle v \psi(0), \Gamma\left(\theta_{t_{j_{r+1}}}\right) U\left(\alpha_{j_{, r}}\right) \Gamma\left(\theta_{t_{j_{r+1}}^{+}}\right) v \psi\left(g \chi_{E_{j, r}}\right)\right\rangle \\
& =\left\langle v \psi(0), U\left(\alpha_{j_{r} r}\right) v \psi\left(\left(\theta_{t_{j_{r+1}}^{+}} g\right) \chi_{\left[0, \alpha_{j, r}\right.}\right)\right\rangle
\end{aligned}
$$




$$
=\|v\|^{2}+\int_{0}^{\alpha_{\jmath}, r}\left\langle v \psi(0), U(\tau) K_{0, g}\left(\tau+t_{j_{r+1}}\right) v \psi\left(\left(\theta_{t_{j_{r+1}}^{+}} g\right) \chi_{\left[0, \alpha_{j}, r\right.}\right)\right\rangle d \tau .
$$

Furthermore

$$
\begin{gathered}
\left|\int_{0}^{\alpha_{j, r}}\left\langle v \psi^{\prime}(0), U(\tau) K_{0, g}\left(\tau+t_{j_{r+1}}\right) v \psi\left(\theta_{t_{j_{r+1}}^{+}} g\right) \chi_{\left[0, \alpha_{j, r}\right)}\right)\right\rangle d \tau \mid \\
\leqq\|v\|^{2} C_{2}\left(\delta(\mathscr{Q})+C_{3} \omega^{\prime}(\mathscr{Q})\right)
\end{gathered}
$$

where $C_{2}=\sup _{0 \leq \tau \leq \alpha_{j, r}}\left\|K_{0, g}\left(\tau+t_{j_{r+1}}\right)\right\|$ is finite by (2.8) and $C_{3}$ is a constant depending only on $g$. The result now follows on combining together all the above inequalities.

By directly analogous arguments to those of Proposition 4.3 of [6], we conclude that $I_{\mathcal{P}}(U, v, g, \xi)$ converges strongly to a unique limit in $h$ as $\delta(\mathscr{P}) \rightarrow 0$. We denote this limit by $I^{T}([a, b], U, v, g, \xi)$.

Proposition 2. For $u, v \in h_{0}, f, g \in \mathcal{S}, \xi, \eta \in A_{c}^{\uparrow}$ and $a, b$ continuity points of $P^{T}$ with $0 \leqq a<b<\infty$ we have,

$$
\begin{aligned}
& \left\langle I^{T}([a, b], U, u, f, \xi), I^{T}([a, b], U, v, g, \eta)\right\rangle \\
& \quad=\int_{a}^{b} e^{-\left\langle f^{s}, g\right\rangle}\langle u, v\rangle\left\langle\psi(f), P^{T}(d s) \psi(g)\right\rangle\langle\xi(s), \eta(s)\rangle .
\end{aligned}
$$

Proof. This proceeds along the same lines as that of Proposition 4.5 in [6], that is approximate both stop time integrals by Riemann sums, use (3.2) and the unitarity of each $U(t)\left(t \in \boldsymbol{R}^{+}\right)$and then let the mesh of the partition tend to zero.

Let $A_{c . r}^{\uparrow}$ denote the subspace of $A^{\uparrow}$ comprising those $\xi \in A_{c}^{\uparrow}$ for which

$$
\int_{0}^{\infty}\|\xi(s)\|^{2}\left\langle\psi(f), P^{T}(d s) \psi(f)\right\rangle<\infty
$$

for all $f \in \mathcal{S}$.

For $\xi \in A_{c, T}^{\uparrow}$ we may now proceed as in [6] and use Proposition 2 to extend the definition of $I^{T}([a, b], U, v, g, \xi)$ to all $a, b \in \boldsymbol{R}^{+} \cup\{\infty\}$ with $0 \leqq a<b \leqq \infty$. The argument is almost exactly the same as that of [6] and we recommend the reader to find the details there.

We define an operator $U(T)$ on the dense subspace of $h$ comprising finite linear combinations of vectors of the form $v \otimes \psi(f)\left(u \in h_{0}, f \in \mathcal{S}\right)$ by linear extension of the prescription

$$
U(T) v \otimes \psi(f)=I^{T}([0, \infty], U, v, f, \xi)
$$

where $\xi \in A_{c, T}^{\hat{r}}$ is given by $\xi(s)=\phi\left(f^{s}\right), s \in \boldsymbol{R}^{+} \cup\{\infty\}$. 
$U(T)$ is clearly well defined. Furthermore, it follows from Proposition 2 that $U(T)$ acts isometrically and thus extends uniquely to an isometry on the whole of $h$. We will also denote this latter operator $U(T)$. We call $U(T)$ the stopped operator associated to the process $U$.

\section{§4. A Stopped Cocycle Relation}

In this section, we will require the stop time $P^{T}$ to be finite and regard the operator $V^{T}$, defined above in $\S 2$, as a unitary isomorphism from $h$ onto $h^{T}$.

We define a new unitary process

$$
\begin{gathered}
U^{T}=\left(U^{T}(t), t \geqq 0\right) \quad \text { on } h^{T} \text { by } \\
U^{T}(t)=V^{T} U(t)\left(V^{T}\right)^{-1} .
\end{gathered}
$$

It is not difficult to establish that $U^{T}$ is the unique solution of the following stochastic differential equation in $h^{T}$ :

$$
\begin{aligned}
& d U^{T}=U^{T}\left(L d A^{T \dagger}+(W-I) d A^{T}-L^{+} W d A^{T}+\left(i H-1 / 2 L^{\dagger} L\right) d t\right) \\
& U^{T}(0)=I .
\end{aligned}
$$

Where $L, H$ and $W$ are as in (2.3) and

$$
\left.\begin{array}{l}
A^{T \#}=V^{T} A^{\#}\left(V^{T}\right)^{-1} \\
\Lambda^{T}=V^{T} \Lambda\left(V^{T}\right)^{-1}
\end{array}\right\} .
$$

Now define the stop time $P^{T+t}$ on $h(t>0)$ by the prescription

$$
\left.\begin{array}{l}
P^{T+t}(E)=0 \quad \text { for } E \in \mathscr{B}([0, t]) \\
P^{T+t}(E)=P^{T}(E-t) \quad \text { for } E \in \mathscr{B}((t, \infty))
\end{array}\right\} .
$$

whence the stop time operator associated to $P^{T+t}$ is $T+t I$. By the construction of $\S 3$, we can associate to the process $U$ the stopped operator $U(T+t)$.

\section{Theorem 3.}

$$
U(T+t)=U(T) U^{T}(t)
$$

for $t \in \boldsymbol{R}^{+}$.

Proof. Let $\mathscr{Q}=\left\{a=t_{0}<t_{1}<\cdots<t_{n}<t_{n+1}=b\right\}$ be a partition by continuity points of $[a, b] \subset[t, \infty)$. We thus obtain a partition $\mathscr{P}^{\prime}=\left\{a-t=s_{0}<s_{1}<\cdots<s_{n}\right.$ $\left.<s_{n+1}=b-t\right\}$ of $[a-t, b-t]$ where each $s_{j}=t_{j}-t(0 \leqq j \leqq n+1)$.

Using (2.6) and the fact that $\Gamma\left(\theta_{\tau}\right) U(t) \Gamma\left(\theta_{\tau}^{+}\right) \in B\left(h^{\tau}\right)$ for $\tau \in \mathbb{R}^{+}$, we obtain for $v \in h_{0}, g \in \mathcal{S}$ 


$$
\begin{aligned}
& \sum_{j=1}^{n} U\left(t_{\jmath+1}\right) P^{T+t}\left(\left[t_{\jmath}, t_{j+1}\right]\right) v \psi\left(g_{t_{j+1}}\right) \psi\left(g^{t_{j+1}}\right) \\
= & \sum_{j=1}^{n} U\left(s_{\jmath+1}+t\right) P^{T}\left(\left[s_{j}, s_{\jmath+1}\right]\right) v \psi\left(g_{s_{\jmath+1}}\right) \psi\left(g^{s_{\jmath+1}}\right) \\
= & \sum_{j=1}^{n} U\left(s_{\jmath+1}\right) \Gamma\left(\theta_{s_{\jmath+1}}\right) U(t) \Gamma\left(\theta_{s_{j+1}}^{+}\right) P^{T}\left(\left[s_{\jmath}, s_{\jmath+1}\right]\right) v \psi\left(g_{s_{j+1}}\right) \psi\left(g^{s_{j+1}}\right) \\
= & \left(\sum_{j=1}^{n} U\left(s_{\jmath+1}\right) P^{T}\left(\left[s_{\jmath}, s_{\jmath+1}\right]\right)\right)\left(\sum_{k=1}^{n} \Gamma\left(\theta_{s_{k+1}}\right) U(t) \Gamma\left(\theta_{s_{k+1}^{+}}\right) \times\right. \\
& \left.P^{T}\left(\left[s_{k}, s_{k+1}\right]\right)\right) v \psi\left(g_{s_{k+1}}\right) \psi\left(g^{s_{k+1}}\right) .
\end{aligned}
$$

The required result now follows on letting $\delta(\mathscr{Q}) \rightarrow 0$ and extending the stop time integrals as in $\S 3$ and [6].

(4.5) clearly generalises the cocycle relation (2.6) in "deterministic" time. We would now like to proceed along the lines of [8] and use (4.5) to construct strongly Markov processes in $h$. However the same arguments [9] that show that the stopped Weyl operators constructed in [6] are not adapted to the algebra $B\left(\mathscr{H}_{T}\right) \otimes I$ will also apply to the operators $U(T)$. This leads us to make the conjecture that the quantum Markov process [8] $\left\{j_{t}, t \in \boldsymbol{R}^{+}\right\}$given by

$$
j_{t}(X)=U(t) X U(t)^{*}
$$

where $X \in B\left(h_{0}\right)$, will not satisfy the strong Markov property.

\section{References}

[1] R.L. Hudson, The Strong Markov Property for Canonical Wiener Processes, J. Funct. Anal. 34, 266 (1979).

[2] D. Applebaum, The Strong Markov Property for Fermion Brownian Motion, J. Funct. Anal. 65, 273 (1986).

[3] C. Barnett and T. J. Lyons, Stopping Non-commutative Processes, Math. Proc. Camb. Phil. Soc. 99, 151 (1986).

[4] P. A. Meyer, Eléments de Probabilités Quantiques, in Séminaire de Probabilités XX ed. J. Azéma, M. Yor (Springer LNM 1204), 186 (1986).

[5] R. L. Hudson and K. R. Parthasarathy, Quantum Ito's Formula and Stochastic Evolutions, Commun. Math. Phys. 93, 301 (1984).

[6] K. R. Parthasarathy and K. B. Sinha, Stop Times in Fock Space Stochastic Calculus, Probability Theory and Related Fields, 75, 317 (1987).

[7] R.L. Hudson and K. R. Parthasarathy, Stochastic Dilations of Uniformly Continuout, Completely Positive Semigroups, Acta Appl. Math. 2, 353 (1984).

[8] A. Frigerio, Covariant Markov Dilations of Quantum Dynamical Semigroups, Publ. RIMS, Kyoto Univ., 21, 657 (1985).

[9] L. Accardi and K. B. Sinha, in preparation. 
